\title{
A Study on the Radiation Hardness of Lead Tungstate Crystals ${ }^{1}$
}

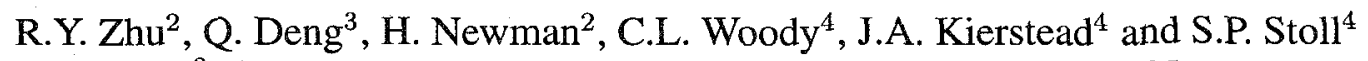 \\ 2 256-48, HEP, California Institute of Technology, Pasadena, CA 91125 \\ ${ }^{3}$ Shanghai Institute of Ceramics, Chinese Academy of Sciences, Shanghai 200050 \\ ${ }^{4}$ Physics Department, Brookhaven National Laboratory, Upton, NY 11973
}

\begin{abstract}
This report presents recent progress of a study on the radiation damage in lead tungstate $\left(\mathrm{PbWO}_{4}\right)$ crystals. The dose rate dependence of radiation damage in $\mathrm{PbWO}_{4}$ has been observed. An optimization of the oxygen compensation through post-growth thermal annealing has led to $\mathrm{PbWO}_{4}$ samples with significantly improved radiation hardness. Front irradiation is found to cause a factor of 2 to 6 times less severe damage than uniform irradiation. Lanthanum doping was found not to be a determining factor for $\mathrm{PbWO}_{4}$ radiation hardness improvement. Finally, a TEM/EDS analysis revealed that the radiation damage in $\mathrm{PbWO}_{4}$ crystals is caused by oxygen vacancies.
\end{abstract}

\section{INTRODUCTION}

Because of its high density and fast decay time, lead tungstate $\left(\mathrm{PbWO}_{4}\right)$ crystals were chosen by the CMS experiment to construct a precision electromagnetic calorimeter at LHC [1]. Our previous studies on $\mathrm{PbWO}_{4}$ samples revealed that $\mathrm{PbWO}_{4}$ crystals suffer from non-negligible radiation damage after a dose as low as $10 \mathrm{rad}$ [2], but the scintillation mechanism of $\mathrm{PbWO}_{4}$ is not affected by the radiation, i.e. the loss of light output is due only to absorption by radiation induced color centers [3]. We also proposed that the damage in $\mathrm{PbWO}_{4}$ is caused by structure related defects, such as oxygen vacancies [2], and the level of the damage may be dose rate dependent because of the damage recovery observed [4].

In this report, we present progress in our understanding on the radiation damage in $\mathrm{PbWO}_{4}$ crystals. Section II describes a dose rate dependence and the result of an optimization of the oxygen compensation, carried out at Shanghai Institute of Ceramics (SIC). A comparison of different irradiation patterns is presented in Section III. Section IV discusses the results of measurements of a preliminary batch of lanthanum (La) doped $\mathrm{PbWO}_{4}$ samples. A microscopic analysis using Transmission Electron Microscopy (TEM) coupled to Energy Dispersion Spectrometry (EDS) is presented in Section V. This work is a part of an ongoing effort aimed at developing radiation hard $\mathrm{PbWO}_{4}$ crystals for CMS at LHC [5].

\footnotetext{
${ }^{1}$ Work at Caltech supported in part by U.S. Department of Energy Grant No. DE-FG03-92-ER40701, and at BNL in part by U.S. Department of Energy Contract No. DE-AC02-CH7600016.
}

\section{DOSE RATE DEPENDENCE AND OPTIMIZATION OF OXYGEN COMPENSATION}

Twelve $\mathrm{PbWO}_{4}$ samples were measured to study the effect of post-growth oxygen compensation on the radiation hardness of $\mathrm{PbWO}_{4}$ crystals. Table 1 lists their dimensions, date of delivery and the conditions used for post-growth thermal annealing.

Table 1

$\mathrm{PbWO}_{4}$ Samples Tested for Oxygen Compensation

\begin{tabular}{|c|c|c|c|}
\hline $\mathrm{I} \mathrm{D}$ & Dimension $(\mathrm{cm})$ & Date & Annealing \\
\hline BGRI 1 & $2.3 \times 5 \times 2.3$ & $1 / 97$ & 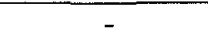 \\
\hline SIC 93 & $2.5 \times 5 \times 2.5$ & $1 / 97$ & - \\
\hline SIC 115-1 & $2 \times 5 \times 2$ & $1 / 97$ & $\mathrm{O}_{2}-1$ \\
\hline SIC 121-1 & $2 \times 5 \times 2$ & $1 / 97$ & $\mathrm{O}_{2}-1$ \\
\hline SIC $117-1$ & $2 \times 5 \times 2$ & $1 / 97$ & $\mathrm{O}_{2}-2$ \\
\hline SIC 122-1 & $2 \times 5 \times 2$ & $1 / 97$ & $\mathrm{O}_{2}-2$ \\
\hline SIC $115-2$ & $2 \times 5 \times 2$ & $1 / 97$ & Air-1 \\
\hline SIC 121-2 & $2 \times 5 \times 2$ & $1 / 97$ & Air-1 \\
\hline SIC 116-2 & $2 \times 5 \times 2$ & $1 / 97$ & Air-2 \\
\hline SIC 117-2 & $2 \times 5 \times 2$ & $1 / 97$ & Air-2 \\
\hline SIC $152-2$ & $2 \times 5 \times 2$ & $8 / 97$ & Optimized $\mathrm{O}_{2}$ \\
\hline SIC 153 & $2 \times 5 \times 2$ & $8 / 97$ & Optimized $\mathrm{O}_{2}$ \\
\hline
\end{tabular}

Samples were prepared in 6 different annealing conditions, ranging from no annealing at all, two kinds of annealing in air: Air- 1 and Air-2, and three kinds of annealing in oxygen: $\mathrm{O}_{2}-1, \mathrm{O}_{2}-2$ and final optimized $\mathrm{O}_{2}$. The difference between different annealing conditions is in the temperature, duration and partial pressure of the oxygen in the oven. In order to test the reproducibility of the procedure, each annealing condition was tested using a pair of crystals cut from different boles. In addition, two samples from the same bole were treated with different annealing conditions (e.g., 115-1 and 115-2 were treated in oxygen and air, respectively). All samples, except BGRI 1, were grown at SIC.

Samples were wrapped with aluminum foil, and were placed at a fixed distance to a $\gamma$-ray source to receive a defined dose rate. Samples were under irradiation all the time, except when measurements were carried out, which typically lasted for about 20 minutes. The absolute light output was measured using a Hamamatsu R2059 PMT with a small ${ }^{137} \mathrm{Cs}$ source placed at the far end of the crystal. The systematic uncertainty of the light output due to temperature variation in the lab is less than $0.3 \%$. The overall systematic uncertainty of the light 


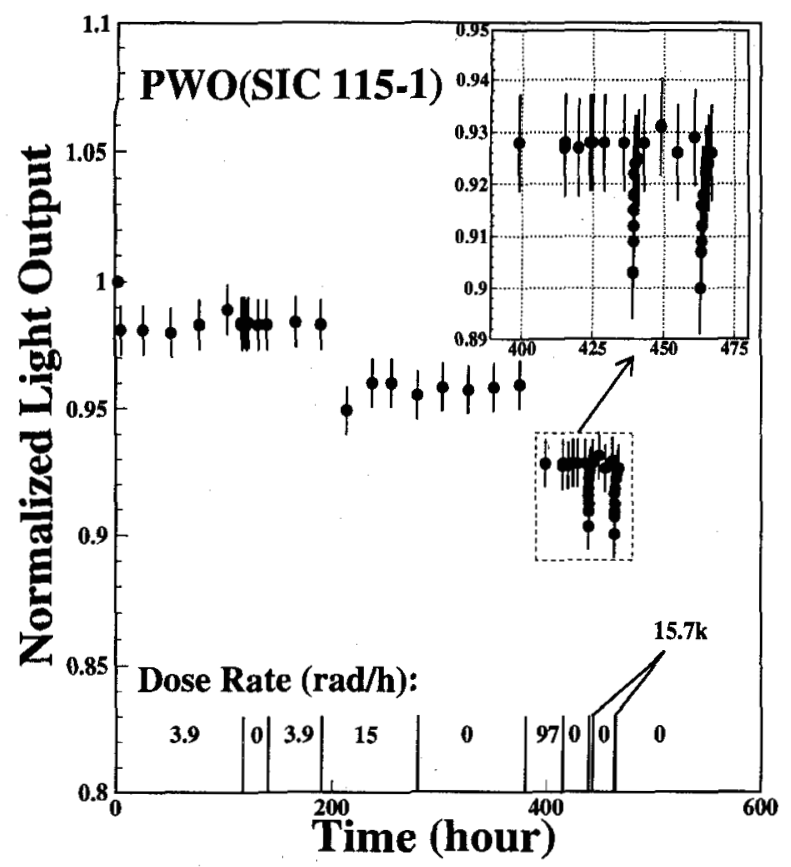

Fig. 1 The normalized light output of sample $115-1$ is shown as a function of time and dose rate.

output measurement is less than $1 \%$ for samples of more than 10 p.e. $/ \mathrm{MeV}$, and is increased to about $2 \%$, when the light output is about 5 p.e./MeV caused by uncertainties in peak finding.

Figure 1 shows the light output normalized to that before irradiation as a function of time for sample 115-1, together with the dose rate. There are periods when the dose rate is shown as zero when a recovery test was carried out. The result shows that the level of $\mathrm{PbWO}_{4}$ damage is dose rate dependent, and that the fast recovery (on the order of a few hours) was observed only when the dose rate was $15.7 \mathrm{krad} / \mathrm{h}$, as shown in the insert. This dose rate dependence is expected according to a simple model of color center kinetics [4]. It should be noted that the typical dose rate expected under full luminosity at LHC is approximately $15 \mathrm{rad} / \mathrm{h}$ in the Barrel of the CMS $\mathrm{PbWO}_{4}$ calorimeter, and up to $500 \mathrm{rad} / \mathrm{h}$ at the Endcaps.

The light output degradation was found to be clearly correlated with the annealing conditions. Figure 2 shows the normalized light output as a function of dose rate. Measurements were done step by step for four dose rates: 15 , $100,480,15.7 \mathrm{k} \mathrm{rad} / \mathrm{h}$. It shows that samples annealed under different conditions have much different behavior. Samples SIC 93 and BGRI 1, which were not annealed, have the worst radiation hardness. Samples annealed in oxygen are more radiation hard than those annealed in air. Samples SIC 152-2 and SIC 153, which were annealed under the optimized oxygen conditions, are the best. This observation suggests that oxygen related defects play a key role in the radiation damage process.

Samples treated under the optimized oxygen annealing are the most radiation hard. Figure 3 shows the normalized light output of sample 153 as a function of integrated dose up to $20 \mathrm{Mrad}$. This sample showed no degradation in light

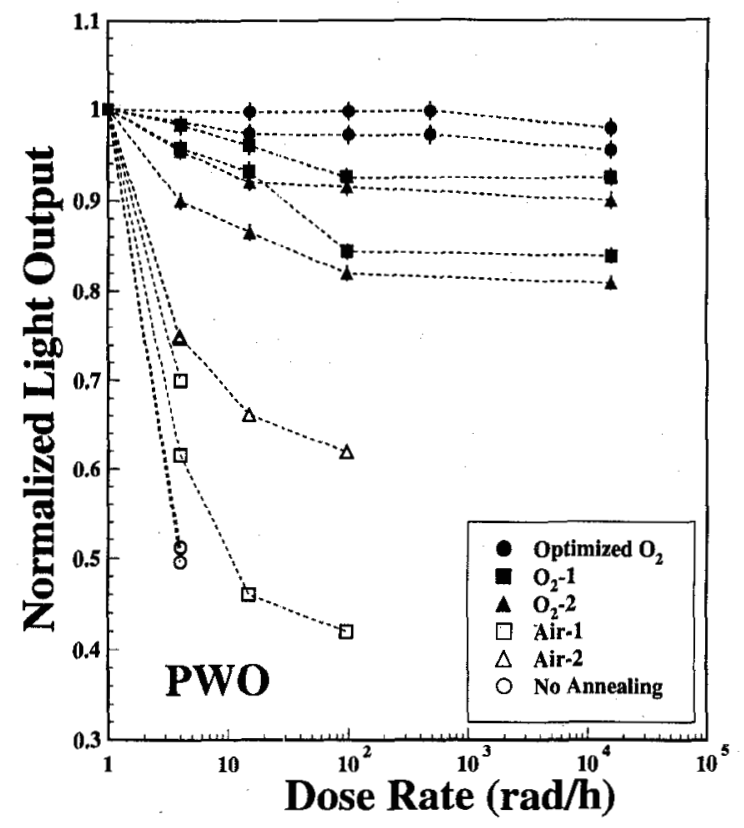

Fig. 2 Normalized light output degradation is shown as a function of the dose rate for sarnples prepared under different annealing conditions.

output under a dose rate below $480 \mathrm{rad} / \mathrm{h}$, and had $\sim 2 \%$ degradation under an extremely high dose rate of $15.7 \mathrm{krad} / \mathrm{h}$. Consistent results were obtained at the Paul Scherre Institute (PSI) irradiation facility for other $\mathrm{PbWO}_{4}$ samples treated with optimized annealing [5]. Samples of this quality certainly satisfy the most stringent radiation hardness requirements for CMS. Further effort, however, must be made to apply this annealing technology to full size crystals.

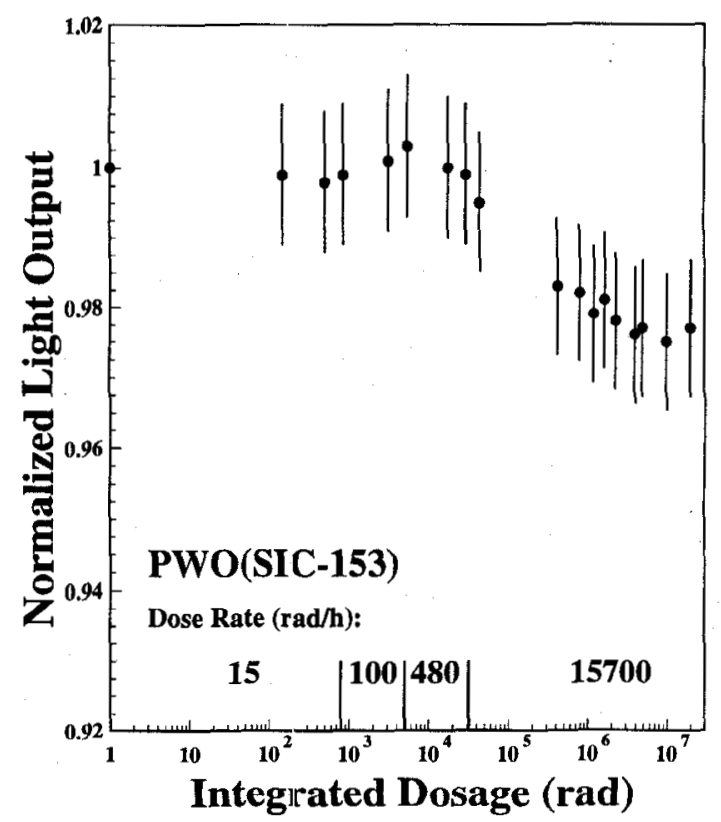

Fig. 3 The normalized light output of sample SIC 153 is shown as a function of integrated dose. 


\section{FRONT VERSUS UNIFORM IRRADIATIONS}

Two most common irradiation patterns are uniform and front irradiations. In front irradiation, the radiation is applied perpendicular to the small end of a long sample. In uniform irradiation, the radiation is applied perpendicular to one long side of the crystal, and uniformity along the length. With a $\gamma$-ray irradiation source, the uniform irradiation exposes the entire volume of the crystal, while the front irradiation only exposes a few centimeters at the front.

Table 2

Samples Tested for Front and Uniform Irradiations

\begin{tabular}{cccc}
\hline \hline ID & Dimension (cm) & Date & Annealing \\
\hline SIC 85 & $2.0 \times 20 \times 2.3$ & $8 / 96$ & $\mathrm{O}_{2}$ \\
SIC 135 & $2.0 \times 23 \times 2.3$ & $5 / 97$ & $\mathrm{O}_{2}$ \\
SIC 136 & $2.0 \times 23 \times 2.3$ & $5 / 97$ & $\mathrm{O}_{2}$ \\
\hline \hline
\end{tabular}

A total of three full size samples were tested, as shown in Table 2. These samples have a tapered shape with small and large ends of 2 and $2.3 \mathrm{~cm}$ square respectively, and a length of 20 to $23 \mathrm{~cm}$. Samples were prepared through our standard process of thermal annealing at $250^{\circ} \mathrm{C}$ for two hours and were irradiated at a fixed dose rate of 15,100 and $480 \mathrm{rad} / \mathrm{h}$. At each dose rate, the light output of these samples were measured until they reached equilibrium, as defined by no change of light output in 24 hours under irradiation. After that, the dose rate was increased to the next higher level. The same procedure was followed for both front and uniform irradiations. We found that the light output degradation under uniform irradiation is a factor of 2 to 6 worse than that of front irradiation. A typical result is shown in Figure 4 for sample 85 . While this sample suffered a damage of $2 \%$ at $15 \mathrm{rad} / \mathrm{h}$ under front irradiation,

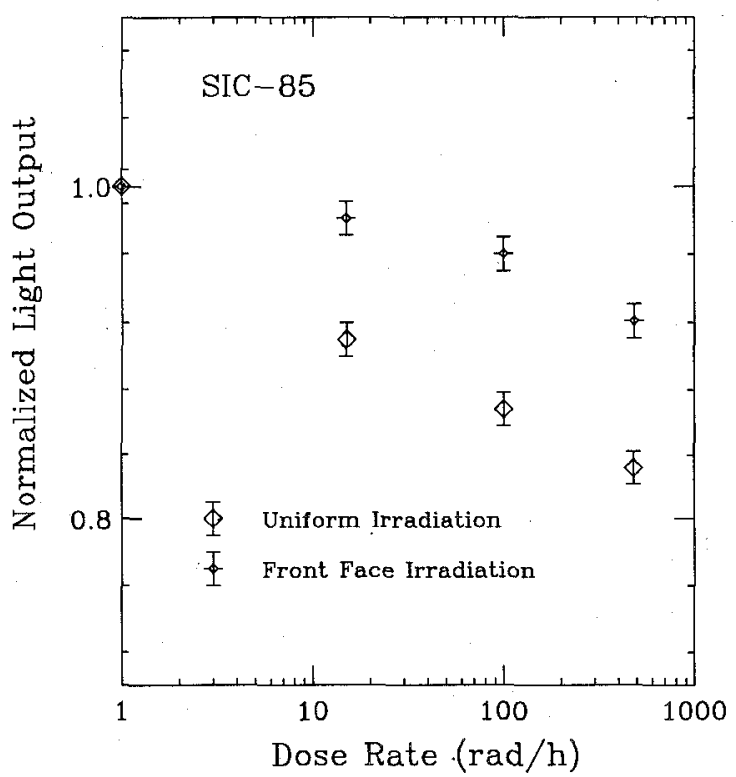

Fig. 4 Light output degradation at equilibrium is shown as a function of dose rate for uniform and front irradiations for sample SIC 85. it was increased to about $8 \%$ under uniform irradiation. The uniform irradiation caused much more severe damage, since color centers were created in the entire body of the crystal.

\section{EFFECT OF LANTHANUM DOPING}

The doping of lanthanum in $\mathrm{PbWO}_{4}$ crystals is known to improve the UV cut-off edge of the transmittance [6]. In the process of developing rad-hard $\mathrm{PbWO}_{4}$ crystals for $\mathrm{CMS}$, we also recognized that lanthanum doping might be an alternative approach to reduce the density of oxygen vacancies. Lanthanum doping is carried out by introducing $\mathrm{La}_{2} \mathrm{O}_{3}$ powder in raw material. This results in one molecule of $\mathrm{La}_{2} \mathrm{O}_{3}$ replacing two $\mathrm{PbO}$ molecules and, consequently, filling one oxygen vacancy. A preliminary batch of lanthanum doped samples were grown at SIC to test the effectiveness of La doping. Table 3 lists the sample ID, dimension, annealing condition and the amount of lanthanum in the raw material. All samples were delivered in June, 1997.

Table 3

Samples Tested for the La Doping

\begin{tabular}{cccc}
\hline \hline $\mathrm{ID}$ & Dimension $(\mathrm{cm})$ & Annealing & La (ppm) \\
\hline SIC L-1 & $2 \times 5 \times 2$ & $\mathrm{O}_{2}$ & 1960 \\
SIC L-5 & $2 \times 5 \times 2$ & - & 168 \\
SIC L-6 & $2 \times 5 \times 2$ & - & 84 \\
\hline \hline
\end{tabular}

We found that $\mathrm{La}$ doping indeed improves the UV cut-off edge of the transmission, confirming the earlier observation by Kobayashi et al. [6]. Figure 5 shows the longitudinal transmission of sample SIC L-5, measured with a Hitachi U-3210 spectrophotometer before irradiation and

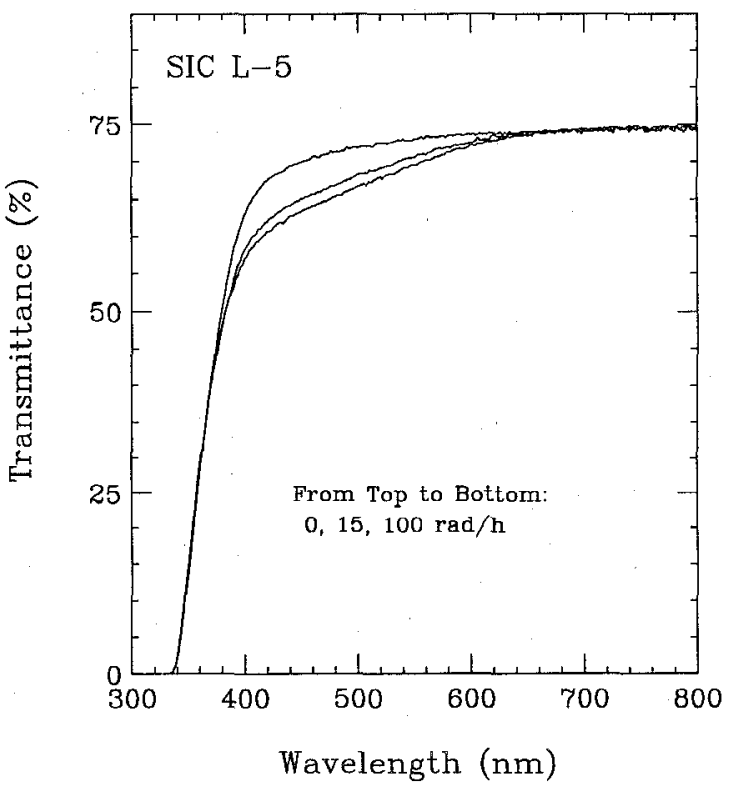

Fig. 5 Transmittance at equilibrium at different dose rates is shown as a function of wavelength for sample SIC L-5. 


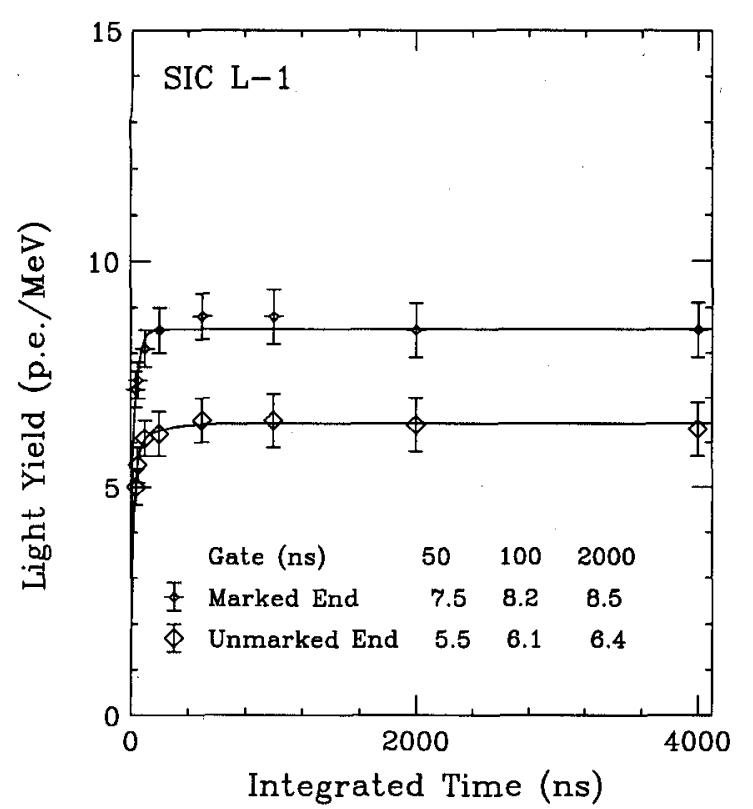

Fig. 6 Light output of sample SIC L-1 is plotted as a function of integration time, showing different amplitudes when different ends of the sample were coupled to the PMT.

at equilibrium at dose rates of 15 and $100 \mathrm{rad} / \mathrm{h}$. A very sharp rising at $340 \mathrm{~nm}$ was observed both before and after irradiation, as compared to samples without $\mathrm{La}$ doping. A radiation-induced absorption band peaked at $420 \mathrm{~nm}$, however, is clearly observed for this sample. The transmittance of the other La doped samples show similar features, indicating that La doping alone does not improve the radiation hardness.

We also found that the peak of the emission spectrum of La doped samples shifted from the green $(500 \mathrm{~nm})$ to the blue $(420 \mathrm{~nm})$, and the light output of these samples is sensitive to the La concentration, indicating that La doping may change the $\mathrm{PbWO}_{4}$ scintillation mechanism. To verify this, the light output was measured as a function of integration time for each sample with two different ends coupled to the PMT. Figure 6 shows the result for sample SIC L-1. While the light output of La doped samples is indeed faster than samples without La doping, it depends on the end coupled to the PMT. Thirty percent more light was observed for sample SIC L-1 after switching the end coupled to the PMT. This highly non-uniform light output was observed in all three La doped samples, but was not observed in any undoped sample.

Assuming La doping affects the $\mathrm{PbWO}_{4}$ scintillation, this non-uniformity may be caused by a non-uniform La concentration in the sample. A Glow Discharge Mass Spectroscopy (GDMS) analysis was carried out at Shiva Technologies West, Inc., California. Table 4 shows the result of the trace element analysis, in ppm by weight, for five pieces of $\mathrm{PbWO}_{4}$ samples: three pieces from the top, middle and bottom portion of sample SIC L6, and two pieces from two ends of another sample L4. As seen from this table, the La concentration is indeed not uniform within the sample. We thus conclude that the origin of this light output non-uniformity is due to very different La concentrations in the sample caused by

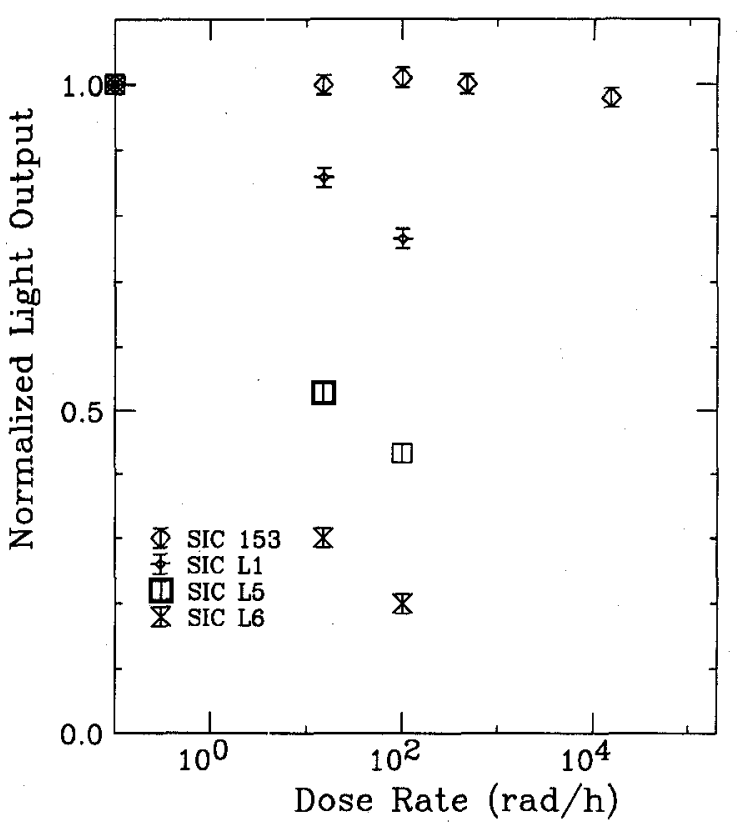

Fig. 7 Normalized light output at equilibrium is shown as a function of dose rate for three La doped samples, and compared to oxygen annealed sample SIC 153.

a segregation coefficient not equal to one. Table 4 also indicates that while impurities of $\mathrm{Na}, \mathrm{K}, \mathrm{Mo}$, As and Eu moved to the top of the crystal during the growth process with a segregation coefficient of less than one, impurities of $\mathrm{Ca}$ and La stayed at the bottom of the crystal with a segregation coefficient larger than one. Note that SIC uses a modified Bridgman method to grow $\mathrm{PbWO}_{4}$ crystals from the bottom up.

Finally, it is concluded that La doping alone does not improve $\mathrm{PbWO}_{4}$ radiation hardness. Figure 7 shows the normalized light output at equilibrium under specific dose rates for these La doped samples, and compared to sample 153 which is treated with optimized oxygen compensation. It is clear that SIC 153 has much better radiation hardness than all of the La doped! samples. Note, however, this batch of La doped samples was preliminary, and further efforts will be made to dope lanthanum more uniformly in $\mathrm{PbWO}_{4}$. As discussed above, La doping indeed improves the transmittance of the crystal, and may help in reducing oxygen vacancies. Therefore, $\mathrm{La}$ and other tri-valent doping studies will continue until a final conclusion is drawn.

\section{Lead Tungstate Damage Mechanism}

Crystal defects, such as oxygen vacancy, are known to cause radiation damage for other oxide scintillators. In BGO, for example, three common radiation induced absorption bands at $2.3,3.0$ and $3.8 \mathrm{eV}$ with consistent widths were found in a series of 24 doped samples [7], indicating defect-related color centers.

In our previous work, we concluded that $\mathrm{PbWO}_{4}$ damage is caused by structure related defects, such as oxygen vacancies [2]. This conclusion was reached based upon material analysis carried out for a batch of $\mathrm{PbWO}_{4}$ crystals grown in Bogoroditsk Techno-Chemical Plant (BTCP), Russia, and SIC. We first did GDMS analysis at Charles Evens \& Associates, California. 
Table 4

Trace Impurity Result (ppmw) Obtained by GDMS Analysis for Five La Doped $\mathrm{PbWO}_{4}$ Samples

\begin{tabular}{lcccccccccc}
\hline \hline $\mathrm{ID}$ & $\mathrm{Na}$ & $\mathrm{Al}$ & $\mathrm{Si}$ & $\mathrm{K}$ & $\mathrm{Ca}$ & $\mathrm{La}$ & $\mathrm{Mo}$ & $\mathrm{As}$ & $\mathrm{TE}$ & Eu \\
\hline $\mathrm{L}-6(\mathrm{~T})$ & 9.1 & 0.2 & 0.3 & 1.4 & 0.8 & 2.7 & 1.3 & 0.93 & 0.2 & 4.3 \\
$\mathrm{~L}-6(\mathrm{M})$ & 2.7 & 0.04 & 0.4 & 0.7 & 2.9 & 38 & 0.92 & 0.19 & 0.01 & 2.1 \\
$\mathrm{~L}-6(\mathrm{~B})$ & 1.9 & 0.1 & 0.2 & 0.5 & 6.2 & 85 & 0.84 & 0.13 & 0.02 & 1.5 \\
\hline $\mathrm{L}-4(\mathrm{~T})$ & 1.4 & 0.4 & 0.5 & 5.9 & 0.05 & 2.8 & 1.8 & 3.9 & 0.2 & 1.2 \\
$\mathrm{~L}-4(\mathrm{~B})$ & 0.2 & 0.7 & 0.6 & 0.4 & 0.38 & 590 & 0.68 & 0.64 & 0.1 & 0.6 \\
\hline \hline
\end{tabular}

A survey of 76 elements, including all of the lanthanides, indicates that there are no obvious correlations between the detected trace impurities and the crystal's susceptibility to radiation damage. This indicates an important role of defects, such as oxygen vacancies, which cannot be determined by GDMS. We then did Particle Induced X-ray Emission (PIXE) and quantitative wavelength dispersive Electron Micro-Probe Analysis (EMPA) at Charles Evans \& Associates, and found that crystals with poor radiation hardness had an abnormal stoichiometric $\mathrm{W} / \mathrm{Pb}$ ratio.

As discussed in Section II, we have observed a significant improvement in the radiation hardness of $\mathrm{PbWO}_{4}$ crystals from oxygen compensation through post-growth thermal annealing in an oxygen-rich atmosphere. This is a pragmatic approach to develop radiation hard $\mathrm{PbWO}_{4}$ crystals, but is not a direct observation of oxygen vacancies in the crystal. To directly observe oxygen vacancies, one must be able to measure the

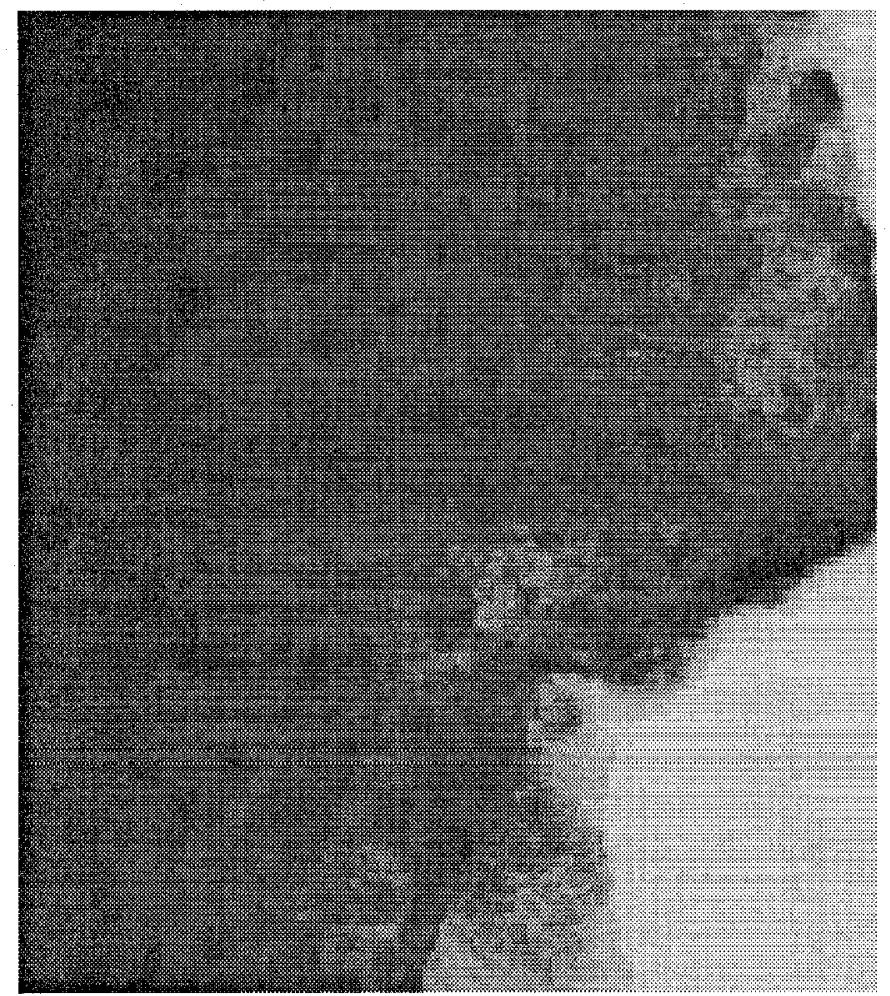

Fig. $8 \mathrm{TEM}$ picture of a $\mathrm{PbWO}_{4}$ sample with poor radiation hardness, showing black spots of $\phi 5-10 \mathrm{~nm}$ related to oxygen vacancies. complete stoichiometric ratio of $\mathrm{Pb}: \mathrm{W}: \mathrm{O}$ in $\mathrm{PbWO}_{4}$ samples. Efforts have been made along this line. X-ray Photoelectron Spectroscopy (XPS) was tried at Charles Evens \& Associates [8]. However, it was found to be very difficult due to the systematic uncertainty in the oxygen analysis. Effort has also be made to directly identify oxygen vacancies by Electron Paramagnetic Resonance (ESR) and Electron-Nuclear Double Resonance (ENDOR) through observing unpaired electrons. However, it too was also difficult to reach a quantitative conclusion.

By using Transmission Electron Microscopy (TEM) coupled to Energy Dispersion Spectrometry (EDS), a localized stoichiometry analysis was possible to identify oxygen vacancies. A TOPCON-002B Scope was first used at $200 \mathrm{kV}$ and $10 \mu \mathrm{A}$. Samples were made into powders of an average grain size of a few $\mu \mathrm{m}$, and then placed on a sustaining

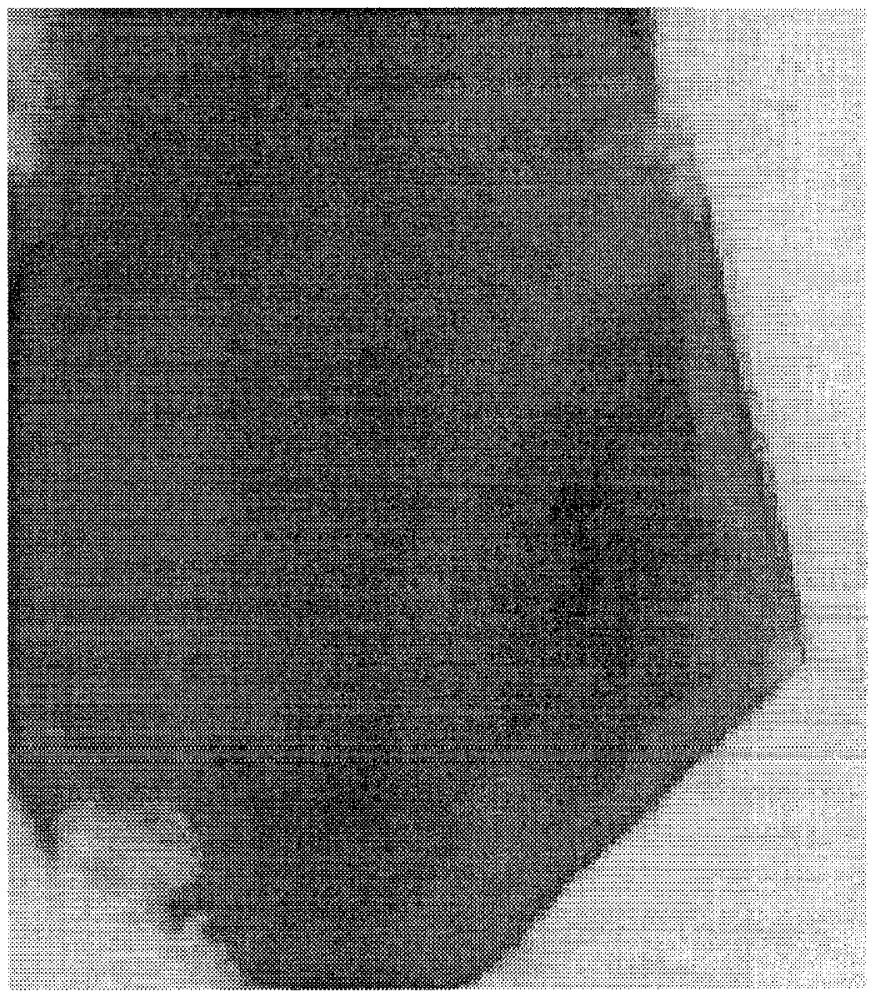

Fig. 9 TEM picture of a $\mathrm{PbWO}_{4}$ crystal with good radiation hardness, showing no black spots. 
membrane. With a spatial resolution of $2 \AA$, the lattice structure of $\mathrm{PbWO}_{4}$ crystals was clearly visible.

Figure 8 shows a TEM picture taken for a sample with poor radiation hardness. Some black spots of a diameter of 5-10 $\mathrm{nm}$ are clearly seen in the picture. On the other hand, samples with good radiation hardness show a stable TEM picture with no black spots (Figure 9).

By employing TEM with an EDS system, a localized stoichiometry analysis was carried out at SIC. The system is a JEOL JEM-2010 scope and a Link ISIS EDS. The spatial resolution of this system allows a localized stoichiometry analysis in a region with a diameter of $0.5 \mathrm{~nm}$ [9]. An as grown sample was first analyzed, and black spots were observed. Points inside and surrounding the black spots were analyzed as well as points far away from the black spots. The uncertainty of the analysis is typically $15 \%$. The result of this analysis is listed in Table 5, where atomic fractions (\%) in these areas are shown. The results show a deviation from the atomic stoichiometry of $\mathrm{O}: \mathrm{W}: \mathrm{Pb}=66: 17: 17$ in the center of the black spots, pointing to a severe deficit of the oxygen component. In the peripheral area, the oxygen deficit is less, but still significant. There is no oxygen deficit observed in the area far away from the black spots. This analysis thus positively identified oxygen vacancies in $\mathrm{PbWO}_{4}$.

Table 5

Atomic Fraction (\%) of $\mathrm{PbWO}_{4}$ Obtained with Localized ( $\phi 0.5 \mathrm{~nm}$ ) Stoichiometry Analysis by TEM/EDS

As Grown Sample

\begin{tabular}{ccccc}
\hline \hline Element & Black Spot & Peripheral & Matrix $_{1}$ & Matrix $_{2}$ \\
\hline $\mathrm{O}$ & 1.5 & 15.8 & 60.8 & 63.2 \\
$\mathrm{~W}$ & 50.8 & 44.3 & 19.6 & 18.4 \\
$\mathrm{~Pb}$ & 47.7 & 39.9 & 19.6 & 18.4 \\
\hline \hline
\end{tabular}

The Same Sample after Oxygen Compensation

\begin{tabular}{ccccc}
\hline \hline Element & Point $_{1}$ & Point $_{2}$ & Point $_{3}$ & Point $_{4}$ \\
\hline $\mathrm{O}$ & 59.0 & 66.4 & 57.4 & 66.7 \\
$\mathrm{~W}$ & 21.0 & 16.5 & 21.3 & 16.8 \\
$\mathrm{~Pb}$ & 20.0 & 17.1 & 21.3 & 16.5 \\
\hline \hline
\end{tabular}

As a comparison, the same sample after oxygen compensation was re-analyzed. No black spots were found. The result of the analysis is also listed in Table 5 . In all randomly selected points, no stoichiometry deviations were observed. This analysis thus clearly identified oxygen vacancies in $\mathrm{PbWO}_{4}$ samples of poor radiation hardness, and confirmed our early conjecture on the $\mathrm{PbWO}_{4}$ radiation damage mechanism.

\section{SUMMARY}

A brief summary of the investigation carried out in this report is listed below.
1. The level of radiation damage in $\mathrm{PbWO}_{4}$ crystals is dose rate dependent - a confirmation of our prediction published in reference [4].

2. The oxygen compensation through post-growth thermal annealing is effective in improving $\mathrm{PbWO}_{4}$ radiation hardness, and has led to $5 \mathrm{~cm} \mathrm{PbWO}_{4}$ samples of significantly improved radiation hardness. The effect of annealing on the radiation hardness of $\mathrm{PbWO}_{4}$ crystals is reproducible.

3. The light output degradation under uniform irradiation is a factor of 2 to 6 worse than that of front irradiation.

4. Investigation of a preliminary batch of La-doped samples indicates that $\mathrm{La}$ doping does improve the UV cut-off edge of the transmission and increases the fast component light output, but does not appear to improve the radiation hardness. The investigation of La doping will continue in order to reach a final conclusion.

5. The $\mathrm{PbWO}_{4}$ damage mechanism is understood as being due to structure related defects, e.g. oxygen vacancies. These defects were clearly identified by a localized stoichiometry analysis using TEM/EDS. This also explains the effectiveness of oxygen compensation in improving the radiation hardness of $\mathrm{PbWO}_{4}$ crystals

\section{ACKNOWLEDGMENTS}

We thank Prof. Z.W. Yin of SIC and Dr. G. Cheng of BGRI for providing $\mathrm{PbWO}_{4}$ samples described in this report. Many inspiring and interesting discussions with Drs. M. Kobayashi, J.Y. Liao, G.Q. Wu and Z.W. Yin are also acknowledged. Drs. F. Nessi and P. Lecomte cross checked the light output measurement described in Section II.

\section{REFERENCES}

[1] Compact Muon Solenoid Technical Proposal, CERN/LHCC 9438, LHCC/P1 (1994).

[2] R.Y. Zhu et al., Nucl. Instr. and Meth. A376 319 (1996); C. Woody et al., in Proceedings of SCINT95 Int'l Conf. Delft, August 1995 and IEEE-NUCL-S V43 (1996) 1585.

[3] R.Y. Zhu et al., in Proc. of the 6th Int. Conf. on Calorimetry in High Energy Physics, ed. A. Antonelli et al., Frascati Physics Series (1996) 577.

[4] R.Y. Zhu, IEEE Trans. Nucl. Sci. NS-44 468 (1997).

[5] See chapter 2 of CMS Electromagnetic Calorimeter Technical Design Report, CERN/LHCC 97-33 and references therein, and papers from P. Lecoq, P. Lecomte and R.Y. Zhu in Proc. of the 7 th Int. Conf. on Calorimetry in High Energy Physics, Tucson, November 1997.

[6] M. Kobayashi et al., Nucl. Instr. and Meth. A399 261 (1997) and KEK Preprint 97-12.

[7] R.Y. Zhu et al., Nucl. instr. and Meth. A302 69 (1991),

[8] C. Lazik of Charles Evans and Associates, private communications.

[9] Z.W. Yin et al., in Proceedings of SCINT97 Int'l Conf., Shanghai, September 1997. 